Article

\title{
Double-Hybrid DFT Functionals for the Condensed Phase: Gaussian and Plane Waves Implementation and Evaluation
}

\author{
Frederick Stein *D, Jürg Hutter *(D) and Vladimir V. Rybkin *(D) \\ Department of Chemistry, University of Zurich, Winterthurerstrasse 190, 8057 Zurich, Switzerland \\ * Correspondence: frederick.stein@chem.uzh.ch (F.S.); hutter@chem.uzh.ch (J.H.); \\ vladimir.rybkin@chem.uzh.ch (V.V.R.)
}

Received: 8 October 2020; Accepted: 29 October 2020; Published: 6 November 2020

\begin{abstract}
Intermolecular interactions play an important role for the understanding of catalysis, biochemistry and pharmacy. Double-hybrid density functionals (DHDFs) combine the proper treatment of short-range interactions of common density functionals with the correct description of long-range interactions of wave-function correlation methods. Up to now, there are only a few benchmark studies available examining the performance of DHDFs in condensed phase. We studied the performance of a small but diverse selection of DHDFs implemented within Gaussian and plane waves formalism on cohesive energies of four representative dispersion interaction dominated crystal structures. We found that the PWRB95 and $\omega$ B97X-2 functionals provide an excellent description of long-ranged interactions in solids. In addition, we identified numerical issues due to the extreme grid dependence of the underlying density functional for PWRB95. The basis set superposition error (BSSE) and convergence with respect to the super cell size are discussed for two different large basis sets.
\end{abstract}

Keywords: density functional theory; double-hybrid functionals; benchmark, wave-function correlation method

\section{Introduction}

Electronic structure calculations for realistic condensed-phase systems are generally more involved than those for molecules. The former include more atoms and are performed under periodic boundary conditions (PBC), implying interactions between periodic images. Therefore, condensed-phase electronic structure modelling often relies on simple approximations. Tight-binding approaches—semiempirical methods, density functional based tight-binding (DFTB)—used to be the work horse in the field. With increased computational power Kohn-Sham density functional theory (KS DFT) [1] became a standard approach. Recently, implementations of wave function theories (WFT) became available, although their application is far from routine.

In DFT, energy is given as unique functional of electron density alone (Hohenberg-Kohn theorem) [2]. Although the exact functional is unknown, several approximation levels are available, often classified as rungs of the Jacob's ladder of accuracy [3]. The most simple approximation includes only local information on the density (local density approximation, LDA) [4-8]. More eleborate theories take more properties of the density into account. Including the density gradient yields generalized-gradient (GGA) approximations (LYP [9], PBE functionals [10]), whereas including the kinetic energy density gives meta-GGA functionals [11-13] (e.g., TPSS functional [14]).

Incorporating a portion of exact exchange (non-local) leads to hybrid functionals (e.g., PBE0 [15], B3LYP [16]). Exact exchange energy is not density-dependent, but is rather a non-local quantity (dependent on the density matrix) borrowed from WFT, viz. from Hartree-Fock (HF) theory $[17,18]$. 
Hence, the term "hybrid" functional means including quantities from WFT, i.e., the Hartree-Fock exchange energy, into DFT functionals. Further examples of this approach are range-separated methods (HSE [19], WB97X [20]) and double-hybrid functionals, the latter can also involve range separation. Whereas hybrid functionals depend on the occupied KS orbitals, double-hybrid functionals include additionally virtual orbitals. They account for electron correlation in both DFT fashion via exchange-correlation functional and WFT fashion via excited determinants. We will refer to WFT methods which include correlation energy as Wave-function correlation (WFC) method.

Double-hybrid functionals [21-26] can potentially take "the best of the two worlds". GGA-, meta-GGA- and hybrid DFT functionals are relatively fast and accurate for covalently and ionically bound systems. However, they intrinsically fail to describe long-range dispersion interactions (which is often coped with by explicit dispersion corrections [27-32] and non-local functionals [33,34]) and strong correlations. WFC methods, on the other hand, inherently include the correct asymptotic $R^{-6}$ behaviour. Their significant disadvantage is the high computational cost: $N^{4}$ scaling and higher in the canonical formulations. Reduced-cost methods allow decreasing the scaling, although with high prefactors. Consequently, the cost of a double-hybrid DFT calculation is defined by the cost of its WFT part. The question may then arise: why not use pure WFT instead of double-hybrid functionals? The answer is that the $N^{4}-N^{5}$ scaling of WFT methods used for double-hybrid functionals (second-order Møller-Plesset perturbation theory, MP2 [35]; random phase approximation, RPA [36]) are relatively crude approximations, and despite capturing long-range interactions they can be outperformed by DFT functionals. Thus, inclusion of electron correlation in WFT and DFT fashion may lead to the improved accuracy of both at moderate price as compared to highly precise WFT approaches, such as coupled-cluster methods [37] scaling as $N^{6}$ and higher.

Most condensed-phase implementations of electronic structure methods are based either on the use of plane waves (PW) or Gaussian basis sets. Plane waves constitute a basis in a strict mathematical sense: they are orthogonal and complete. In PW basis DFT and correlation energies converge systematically with basis size [38]. However, due to the fact that PW do not reflect the character of chemical bonding, a larger number of basis functions is needed for accurate calculations, which is detrimental for calculations with WFC methods as virtual space becomes huge. Since atom-centered Gaussian functions reasonably approximate atomic orbitals, good accuracy can be achieved with compact basis sets, i.e., at a lower computational price, especially for DFT. WFC energies are more sensitive to basis set size and exhibit slow convergence with basis set size [39], especially for long-range dispersion interactions [40]. WFC methods and DHDFs are available for PW basis sets in VASP [38,41], for Gaussian basis sets in CP2K [42], CRYSTAL [43] and GAMESS (US) [44] and for Slater type basis sets in ADF [45].

\section{Theoretical Background}

In the following, $a, b, \ldots$ are virtual orbital indices, $i, j, \ldots$ occupied orbital indices, $p, q, \ldots$ general orbital indices, and $P, Q, \ldots$ auxiliary function indices. In DFT, the total energy is given as a functional of the total ground-state density $n(\vec{r})$ :

$$
E_{D F T}[n]=T_{0}[n]+E_{n e}[n]+E_{H}[n]+E_{X C}[n],
$$

where $E_{D F T}[n]$ is the total energy functional, $T_{0}[n]$ is the kinetic energy of a reference system of non-interacting electrons, $E_{n e}[n]$ is the nuclei-electron interaction energy, $E_{H}[n]$ is the Hartree energy describing the classical electron-electron interaction energy, and $E_{X C}[n]$ is the exchange-correlation energy describing the quantum mechanical contributions of the electron-electron interaction. The ground-state density is expressed in terms of orbital functions $\psi_{i}(\vec{r})$

$$
n(\vec{r})=\sum_{i}\left|\psi_{i}(\vec{r})\right|^{2}
$$


where $i$ runs over all occupied orbitals. The orbital functions fulfill the orthonormality constraint:

$$
\int d^{3} r \psi_{i}^{*}(\vec{r}) \psi_{j}(\vec{r})=\delta_{i j}
$$

with the Kronecker delta $\delta_{i j}$. The orbitals are solutions of the Kohn-Sham (KS) equation:

$$
\left(-\frac{\Delta}{2}+v_{n e}(\vec{r})+v_{H}[n](\vec{r})+v_{X C}[n](\vec{r})\right) \psi_{i}(\vec{r})=\epsilon_{i} \psi_{i}(\vec{r})
$$

with the potential arising from the nuclei $v_{n e}(\vec{r})$, the Hartree potential $v_{H}[n](\vec{r})$, the exchangecorrelation $(\mathrm{XC})$ potential $v_{\mathrm{XC}}[n](\vec{r})$, and the orbital energy $\epsilon_{i}$ of orbital $i$. In this article, we will consider Gaussian functions centered at the atoms only.

Because the total energy functional is not known explicitly in terms of the ground-state density, we rely on approximations of the $\mathrm{XC}$ functional. These approximate energy functionals are given as integrals of a function explicitly depending on the ground-state density, its gradient and its Laplacian. For convenience, the $\mathrm{XC}$ functional is split into an exchange functional $E_{X}[n]$ and a correlation functional $E_{C}[n]$.

The more complex hybrid density functionals (HDFs) [16] include explicit information of the occupied orbitals. They modify the exchange functional by including a certain amount $\alpha_{X, H F}$ of Hartree-Fock (HF) exchange $E_{X, H F}[n]$ providing the exchange functional

$$
E_{X, h y b r i d}=\alpha_{X, H F} E_{X, H F}[n]+\alpha_{X, D F T} E_{X, D F T}[n] .
$$

We introduce the amount $\alpha_{X, D F T}$ of DFT exchange $E_{X, D F T}[n]$ to reflect that the DFT exchange functional is an already known GGA or meta-GGA functional (compare [15,16]). Using the Mulliken notation (chemists' notation) for electron repulsion integrals

$$
(p q \mid r s)=\int d^{3} r \int d^{3} r^{\prime} \frac{\phi_{p}^{*}(\vec{r}) \phi_{q}(\vec{r}) \phi_{r}^{*}\left(\vec{r}^{\prime}\right) \phi_{s}\left(\vec{r}^{\prime}\right)}{\left|\vec{r}-\vec{r}^{\prime}\right|}
$$

the HF exchange energy can be written as

$$
E_{X, H F}=-\frac{1}{2} \sum_{i j}(i j \mid j i)
$$

Non-HDFs suffer from self-interaction errors [46]. These are reduced in HDFs but usually not fully cancelled since $\alpha_{X, H F} \neq 1$ in general case. This self-interaction error results in erroneous description of charge-separation processes and transition states. But even hybrid methods and HF lack a reasonable description of dispersion interactions decaying like $R^{-6}$ with $R$ being a measure of charge separation.

For increased flexibility, we can further split the exchange functional in a long-range and a short-range functional and describe both with a given mixture of HF theory and DFT resulting in range-separated HDFs [19].

The highest flexibility is achieved by including virtual orbitals $\psi_{a}(\vec{r})$. Double-hybrid density functionals (DHDFs) are HDFs in which the correlation functional is composed of a mixture of a DFT correlation functional $E_{C, D F T}[n]$ with ratio $\alpha_{C, D F T}$ and correlation energy $E_{C, W F T}[n]$ of a WFC method with ratio $\alpha_{C, W F T}$ providing a functional

$$
E_{C, \text { double-hybrid }}=\alpha_{C, W F T} E_{C, W F T}[n]+\alpha_{C, D F T} E_{C, D F T}[n] .
$$

Because WFC methods are computationally more demanding than HDFs or standard DFT functionals, most DHDFs exploit the MP2 theory, the SOS-MP2 theory, or the RPA method. 
The correlation energy within the MP2 theory for closed-shell systems is

$$
E_{C, M P 2}=\sum_{i j a b} \frac{(i a \mid j b)[2(i a \mid j b)-(i b \mid j a)]}{\epsilon_{i}+\epsilon_{j}-\epsilon_{a}-\epsilon_{b}} .
$$

The computationally most expensive step of the MP2 method is given by the transformation of the electron interaction integrals from atom orbital basis to molecular orbital basis leading to a $\mathcal{O}\left(N^{5}\right)$ scaling with $N$ being a measure of system size. The prefactor can be reduced by the resolution-of-the-identity (RI) approach introducing an auxiliary basis in which densities are expanded giving the equation

$$
(p q \mid r s)=\sum_{P} B_{P}^{p q} B_{P}^{r s}
$$

with

$$
\begin{aligned}
B_{P}^{i a} & =\sum_{Q}(p q \mid Q)(Q \mid P)^{-1 / 2} \\
(p q \mid P) & =\int d^{3} r \int d^{3} r^{\prime} \frac{\phi_{p}^{*}(\vec{r}) \phi_{q}(\vec{r}) \phi_{P}\left(\vec{r}^{\prime}\right)}{\left|\vec{r}-\vec{r}^{\prime}\right|} \\
(P \mid Q) & =\int d^{3} r \int d^{3} r^{\prime} \frac{\phi_{P}(\vec{r}) \phi_{Q}\left(\vec{r}^{\prime}\right)}{\left|\vec{r}-\vec{r}^{\prime}\right|} .
\end{aligned}
$$

This method is called RI-MP2 [47,48].

A simplified version of the RI-MP2 method is the Scaled-Opposite-Spin(SOS)-MP2 method [49] given by

$$
E_{C, S O S-M P 2}=-\int_{0}^{\infty} d \tau \operatorname{Tr}\left(\mathbf{Q}_{S O S-M P 2}(\tau) \mathbf{Q}_{S O S-M P 2}^{T}(\tau)\right)
$$

with

$$
\left(\mathbf{Q}_{S O S-M P 2}(\tau)\right)_{P Q}=\sum_{i a} B_{P}^{i a} e^{\tau\left(\epsilon_{i}-\epsilon_{a}\right)} B_{Q}^{i a} .
$$

The integration is carried out numerically using a Minimax quadrature. The RI-SOS-MP2 method scales like $\mathcal{O}\left(N^{4}\right)$.

Another correlation method with increasing popularity is the Random Phase Approximation (RPA) method [50,51] within the RI approximation

$$
E_{C, R P A}=\frac{1}{2} \int_{0}^{\infty} \frac{d \omega}{2 \pi} \operatorname{Tr}\left(\ln \left(1+\mathbf{Q}_{R P A}(\omega)\right)-\mathbf{Q}_{R P A}(\omega)\right)
$$

with

$$
\mathbf{Q}_{R P A}(\omega)=2 \sum_{i a} B_{P}^{i a} \frac{\epsilon_{a}-\epsilon_{i}}{\omega^{2}+\left(\epsilon_{a}-\epsilon_{i}\right)^{2}} B_{Q}^{i a} .
$$

RI-RPA scales like $\mathcal{O}\left(N^{4}\right)$. As with the RI-SOS-MP2 method, the integration is carried out numerically using a Clenshaw-Curtis grid [52] or a Minimax grid [53,54].

All WFC methods and all DHDFs correctly reproduce the $R^{-6}$ energy behaviour of long-range interactions. Comparable to range-separated HDFs, there are DHDFs with range-separated exchange functionals like the $\omega$ B97X-2 functional [55]. Further, there are DHDFs with range-separated correlation functionals [56]. In this article, we will not focus on DHDFs with range-separated correlation functionals and refer to the literature $[40,57-60]$ for more details.

\section{Computational Details}

\subsection{Gaussian and Plane Waves Method (GPW) and Integral Evaluation}

The Gaussian and plane waves method (GPW) [61] allows for efficient periodic calculations with Gaussian basis sets using a dual representation of the electronic density and molecular orbitals. It assumes the use of a primary Gaussian basis for the expansion of matrix quantities (density matrix, 
KS matrix) and an auxiliary plane waves (PW) basis for the evaluation of the Hartree potential and the numerical integration of density functionals. To converge GPW calculations, one has to pay attention to both the size and quality of the Gaussian basis and the energy cutoff for the PWs. In the current implementation, GPW is used for the calculation of the Hartree potential, XC functionals, and two and three center integrals necessary for the RI-MP2 and RI-RPA methods. Exchange integrals are computed analytically using a truncated Coulomb potential [62].

\subsection{Test Systems}

Because we are interested in the description of intermolecular interactions, we are testing the functionals on molecular crystals $(\mathrm{NH} 3, \mathrm{HCN})$ and rare-gase crystals $(\mathrm{Ar}, \mathrm{Ne})$ which have been studied by Sansone et al. [63]. Structural information of the unit cells are summarized in Table 1. In case of molecular solids, we were using structures reoptimized at the B3LYP-D* level [64].

Molecular crystals represent systems with a mixture of covalent bonding and dispersion interactions. $\mathrm{NH}_{3}$ and $\mathrm{HCN}$ crystals additionally contain hydrogen bonds which are crucial for the discription of proteins. In contrast to that, there are only dispersion interactions within the rare-gas crystals. This results in low cohesive energies and the need for well-balanced functionals.

Table 1. Structural information about the bulk structures used in this study. $n_{f u}$ is the number of formula units per unit cell. References for the geometrical information of the respective system are provided in the last column. Please note that there was a mistake in the cell parameters of $\mathrm{CO}_{2}$ provided in reference [63].

\begin{tabular}{cccc}
\hline System & $\boldsymbol{a} ; \boldsymbol{b} ; \boldsymbol{c}(\AA \mathbf{\AA})$ & $\boldsymbol{n}_{\boldsymbol{f} \boldsymbol{u}}$ & References \\
\hline $\mathrm{NH}_{3}$ & 5.048 & 4 & {$[65]$} \\
$\mathrm{HCN}$ & $4.13 ; 4.85 ; 4.34$ & 2 & {$[66]$} \\
$\mathrm{Ne}$ & 4.464 & 4 & {$[67-69]$} \\
$\mathrm{Ar}$ & 5.300 & 4 & {$[70,71]$} \\
\hline
\end{tabular}

\subsection{Parameters of the Calculations}

All calculations have been carried using a development version 8.0 of CP2K [42]. To ensure convergence with respect to the density cutoff, we were using high cutoffs of $1500 \mathrm{Ry}$ for all RPA and MP2 calculations, $4000 \mathrm{Ry}$ for remaining calculations of the molecular crystals $\mathrm{NH}_{3}$ and $\mathrm{HCN}$, and 10,000 Ry for the rare-gas crystals Ar and $\mathrm{Ne}$ (see Section 4.1 for more details) and a relative cutoff of $50 \mathrm{Ry}$. For the rare-gas crystals, we set the parameters EPS_DEFAULT, EPS_PGF_ORB, EPS_SCF, and EPS_SCHWARZ in the HF section to $10^{-30}, 10^{-50}, 10^{-5}$, and $10^{-10}$, respectively, for the molecular crystals, we were using for the same parameters $10^{-20}, 10^{-40}, 10^{-5}$, and $10^{-9}$, respectively (see the CP2K manual for the meaning of these parameters). HF calculations for the bulk systems were using a truncated Coulomb potential with a cutoff radius of roughly half the super cell size. All densities have been smoothed using the NN10 method.

RI-MP2, RI-SOS-MP2 and RI-RPA calculations have been carried out using the GPW method to determine all integrals with a primary cutoff of $300 R y$ and a relative cutoff of $50 R y$. We have exploited an 8-point minimax grid for all RI-RPA and RI-SOS-MP2 calculations.

\subsection{Choice of Functionals and Implementation}

We carried out calculations at the PBE [10], $\omega$ B97M-V [72], $\omega$ B97X-2 [55], PW6B95 [73], PWRB95 [74], SOS-PBE0-2 [75], RI-MP2 [35,47] and RI-RPA [51] levels of theory. PBE and RI-MP2 are used to compare differences between valence-only calculations of our valence-only calculations and the all-electron calculations of Sansone et al. [63]. PW6B95 is a meta-hybrid functional which performed best for weakly interacting systems with more pronounced dispersion interactions. PWRB95 is its RPA-based DHDF. $\omega$ B97M-V is a dispersion-corrected range-separated meta-hybrid functional. $\omega \mathrm{B} 97 \mathrm{X}-2$ is an MP2-based DHDF with range-separated exchange functional. SOS-PBE0-2 
is a RI-SOS-MP2-based DHDF. With this choice, we cover a large variety of different flavours of meta-hybrid and DHDF theories. Due to very high computational cost, we have restricted ourselves to this small, but representative set of functionals: one for each flavour of DHDFs and a corresponding HDF.

PBE calculations have been carried out using the CP2K implementation of PBE. For the $\omega$ B97M-V, and the PW6B95 functionals, we exploited the implementations of the LibXC library [76], version 4.3.4. Since the VV10 dispersion correction is not available in CP2K, we relied on the rVV10 correction and the parametrization suggested by Mardirossian et al. [77]. For the $\omega$ B97X-2 and PWRB95 functionals, we implemented the required parameter sets into the LibXC library.

\subsection{Basis Sets and Pseudopotentials}

The MP2 and RPA implementations within CP2K rely on a pseudopotential (PP) approach with Goedecker-Teter-Hutter PPs [78]. For the PBE functional, we used PPs optimized for PBE, for RPA and MP2 calculations, we were using PPs optimized for HF whereas for both HDFs and all DHDFs, we utilized PPs optimized for the PBE0 functional. All PPs have been taken from the Github repository of Jürg Hutter [79].

Correlation-consistent primary basis sets and suitable auxiliary basis sets of double zeta (DZ) and triple zeta (TZ) quality for the elements C, H, N and O have been taken from Del Ben et al. [80] We have optimized appropriate correlation-consistent primary and auxiliary basis sets of the same qualities for Ne and Ar using the polarization functions of the respective Dunning basis sets $[48,81,82]$. All PPs and primary and auxiliary basis sets are compiled in the Appendxes A-C.

\subsection{Cohesive Energies and Basis Set Superposition Error}

To determine total energies per formula unit, we carried out calculations of $2 \times 2 \times 2,3 \times 3 \times 3$ and $4 \times 4 \times 4$ supercells of all given unit cells and used a linear fit of the total energy per formula unit against the inverse of the cell volume.

Because calculations of cohesive energies usually suffer from basis set superposition errors (BSSE), we perform a counterpoise correction [83]. The BSSE-free cohesive energies $E_{c o h}$ are calculated according to

$$
E_{c o h}=E_{b u l k}-E_{m o l+g h o s t, b u l k}+E_{m o l, b u l k}-E_{m o l, g a s}
$$

with the total bulk energy per formula unit extrapolated to infinite cell volume $E_{b u l k}$, the energy of the molecule with ghost atoms $E_{m o l+g h o s t, b u l k}$, the energy of the molecule using the bulk geometry $E_{m o l, b u l k}$, and the total energy of the molecule using an optimized gas phase structure $E_{\text {mol,gas }}$. For $\mathrm{Ar}$ and $\mathrm{Ne}$, we trivially have $E_{\text {mol,bulk }}=E_{\text {mol,gas }}$.

The corresponding BSSE is given by

$$
\Delta E_{B S S E}=E_{m o l+g h o s t, b u l k}-E_{m o l, b u l k} .
$$

For the BSSE calculations, we took the crystalline structures, chose one molecule (or atom for Ar and $\mathrm{Ne}$ ) surrounded by all ghost atoms within a $3 \times 3 \times 3$ supercell.

\section{Results}

\subsection{General Remarks}

We found the convergence of total energies of meta-HDFs PW6B95 and PWRB95 requires very tight energy cutoffs for the auxiliary PW basis of at least $4000 \mathrm{Ry}$. In contrast to that, calculations with the other meta-HDFs in our benchmark study, $\omega \mathrm{B} 97 \mathrm{M}-\mathrm{V}$, provided reasonable results with a cutoff of only $1200 \mathrm{Ry}$. Because the basis functions for the elements argon and neon are more localized 
than those for hydrogen, carbon and nitrogen, higher cutoffs for the noble gases were needed for an adequate representation of the basis functions of these elements on the grid.

It is well-known that GGA functionals and especially meta-GGA functionals require very tight integration grids for convergence and thus accurate results. Such cutoffs reflect numerical issues and the need for very fine integration grids when using the PW6B95 and PWRB95 functionals. Such grids are not necessary for the $\omega \mathrm{B} 97 \mathrm{M}-\mathrm{V}$ functional which has been optimized with coarser integration grids in mind [72]. Thus, energy differences converged faster with $\omega$ B97M-V and PBE. Nevertheless, the total energies were not converged. To remove any possible problems due to incomplete convergence with respect to cutoffs, we utilized unusually high cutoffs for all density functionals.

Furthermore, we have found convergence problems with the PW6B95 and PWRB95 functionals, which can be resolved with density smoothing. Unfortunately, in some cases an increase of the energy cutoff for the density resulted in SCF convergence issues which could not be resolved with tighter filter thresholds. Nevertheless, we were able to achieve convergence by restarting the calculations with a higher cutoff starting from the converged SCF results with a lower cutoff. This was not possible for argon, where we exploited a cutoff of 4000 Ry for the PW6B95 and PWRB95 functional. Thus, some numbers for the PW6B95 and PWRB95 functionals are not fully converged with respect to the density cutoff.

Due to the higher computational costs, we have not carried out calculations of the $4 \times 4 \times 4$ supercells on the TZ level.

All cohesive energies are compiled in Tables 2 and 3.

Table 2. Cohesion energies $E_{c o h}$ and absolute relative error with respect to experimental results for all considered methods and systems in $\mathrm{kJ} / \mathrm{mol}$ exploiting basis sets of DZ quality. The statistical indicators are the mean absolute error (MAE) and the mean absolute relative error (MARE). ${ }^{a}$ This work. ${ }^{b}$ Values by Sansonse et al. [63]. ${ }^{c}$ Experimental values [68,71,84], corrected for zero-point energy (ZPE) and thermal effects at $298 \mathrm{~K}[63,85]$.

\begin{tabular}{lcccccccccc}
\hline Functional & $E_{\text {coh }}(\mathrm{Ne})$ & $\%$ & $E_{\text {coh }}(\mathrm{Ar})$ & $\%$ & $E_{\text {coh }}\left(\mathrm{NH}_{3}\right)$ & $\%$ & $E_{\text {coh }}(\mathrm{HCN})$ & $\%$ & MAE & MARE \\
\hline $\mathrm{PBE}^{a}$ & -2.92 & 48 & 2.47 & 131 & -39.9 & 9 & -29.0 & 32 & 7.09 & 0.55 \\
$\mathrm{PBE}^{b}$ & -0.27 & 86 & 2.36 & 130 & -26.9 & 25 & -28.0 & 34 & 8.95 & 0.69 \\
$\mathrm{RPA}^{a}$ & 0.82 & 141 & 4.61 & 159 & -19.0 & 47 & -28.1 & 33 & 11.7 & 0.96 \\
$\mathrm{MP2}^{a}$ & 0.83 & 141 & 2.95 & 138 & -27.6 & 23 & -35.2 & 17 & 7.40 & 0.80 \\
$\mathrm{MP2}^{b}$ & 0.22 & 111 & 3.13 & 140 & -24.2 & 33 & -31.7 & 25 & 9.01 & 0.78 \\
$\omega$ B97M-V $^{a}$ & -2.65 & 34 & -8.30 & 7 & -41.2 & 13 & -58.0 & 36 & 5.37 & 0.23 \\
PW6B95 $^{a}$ & -1.99 & 1 & -0.03 & 99 & -27.1 & 25 & -33.8 & 20 & 6.43 & 0.37 \\
PWRB95 $^{a}$ & -2.29 & 16 & -4.15 & 46 & -34.8 & 4 & -40.3 & 5 & 1.91 & 0.18 \\
$\omega$ B97X-2 $^{a}$ & -0.43 & 78 & -3.60 & 53 & -35.2 & 3 & -42.3 & 0 & 1.77 & 0.34 \\
SOS-PBE0-2 $^{a}$ & 0.57 & 128 & 2.76 & 135 & -25.0 & 31 & -31.0 & 27 & 8.98 & 0.81 \\
Expt. $^{c}$ & -1.97 & & -7.73 & & -36.3 & & -42.6 & & & \\
\hline
\end{tabular}

Table 3. Same as Table 2, but with basis sets of TZ quality. ${ }^{b}$ exploits basis sets of augmented DZ quality.

\begin{tabular}{|c|c|c|c|c|c|c|c|c|c|c|}
\hline Functional & $E_{c o h}(\mathrm{Ne})$ & $\%$ & $E_{c o h}(A r)$ & $\%$ & $E_{c o h}\left(\mathrm{NH}_{3}\right)$ & $\%$ & $E_{c o h}(H C N)$ & $\%$ & MAE & MARE \\
\hline $\mathrm{PBE}^{a}$ & -1.22 & 37 & 0.49 & 106 & -28.5 & 21 & -29.5 & 30 & 7.47 & 0.49 \\
\hline $\mathrm{PBE}^{b}$ & -0.40 & 79 & 0.42 & 105 & -26.2 & 27 & -29.7 & 30 & 8.18 & 0.61 \\
\hline $\mathrm{RPA}^{a}$ & 0.32 & 116 & 0.44 & 105 & -24.1 & 33 & -34.9 & 17 & 7.58 & 0.68 \\
\hline $\mathrm{MP}^{a}{ }^{a}$ & 0.05 & 102 & -4.48 & 42 & -34.4 & 5 & -43.9 & 3 & 2.13 & 0.38 \\
\hline $\mathrm{MP} 2^{b}$ & -1.10 & 44 & -6.45 & 16 & -31.8 & 12 & -41.4 & 2 & 1.96 & 0.19 \\
\hline$\omega \mathrm{B} 97 \mathrm{M}-\mathrm{V}^{a}$ & -3.51 & 78 & -9.28 & 20 & -39.5 & 8 & -50.9 & 19 & 3.65 & 0.32 \\
\hline $\mathrm{PW} 6 \mathrm{~B} 95^{a}$ & -2.31 & 17 & -1.95 & 74 & -25.6 & 29 & -32.6 & 23 & 6.72 & 0.36 \\
\hline PWRB95 $^{a}$ & -2.67 & 35 & -3.10 & 59 & -36.1 & 0 & -44.0 & 3 & 1.73 & 0.25 \\
\hline$\omega \mathrm{B} 97 \mathrm{X}-2^{a}$ & -2.59 & 31 & -8.15 & 5 & -38.6 & 6 & -43.0 & 0 & 0.93 & 0.11 \\
\hline SOS-PBE0-2 ${ }^{a}$ & 0.10 & 105 & -0.12 & 98 & -26.4 & 27 & -33.0 & 22 & 7.28 & 0.63 \\
\hline Expt. $^{c}$ & -1.97 & & -7.73 & & -36.3 & & -42.6 & & & \\
\hline
\end{tabular}




\subsection{Convergence with Respect to Super Cell Size}

In Figure 1, we compiled the differences in total energies per formula unit relative to the extrapolated total energies. In general, we expect the total energies to decrease with increasing supercell size and the extrapolated value is a lower bound for the total energies of the super cells. Our results show exactly this behaviour.

Total Energies per Formula Unit Relative to the Extrapolated Values in $\mathrm{kJ} / \mathrm{mol}$
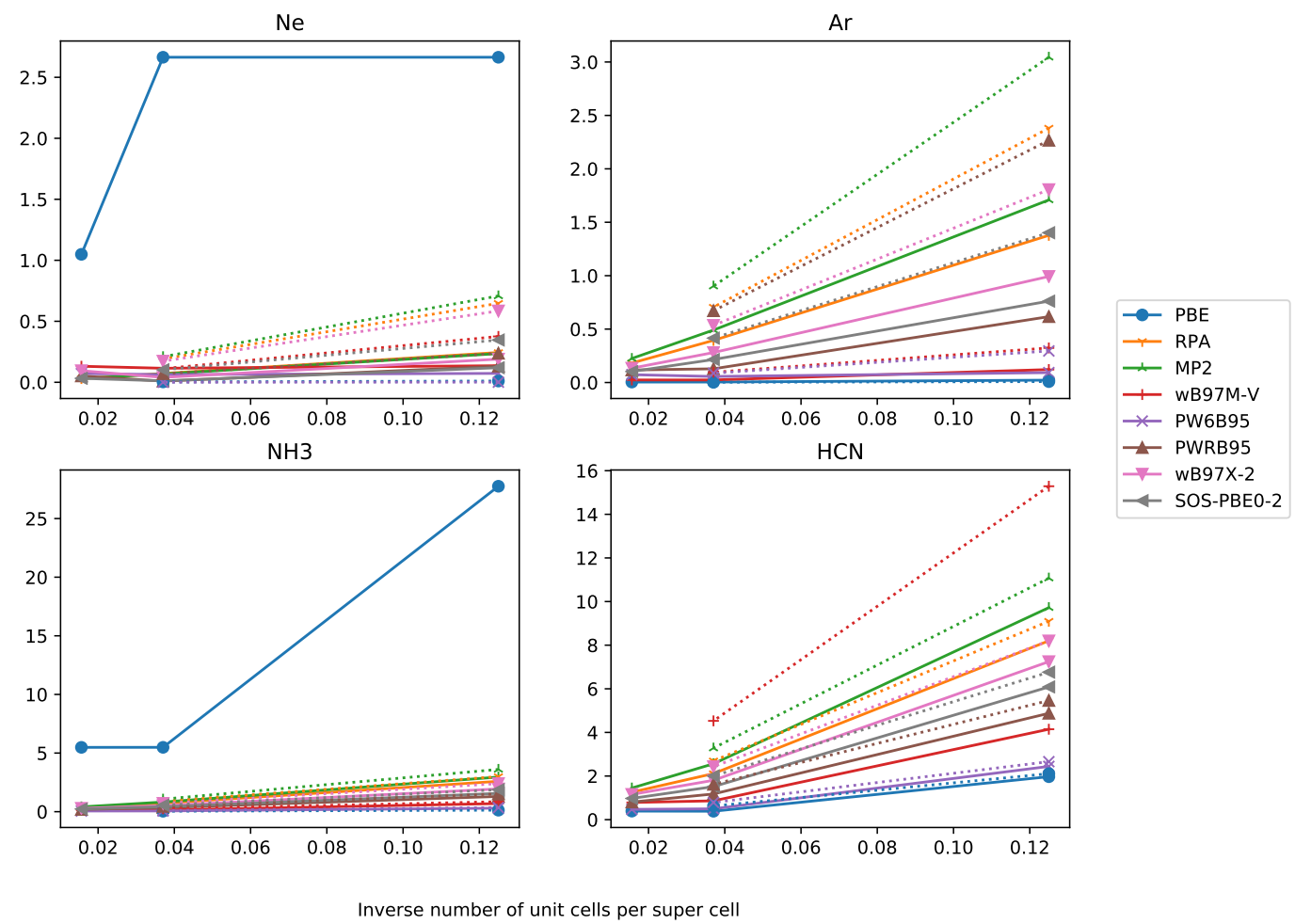

Figure 1. Total energies per formula unit relative to extrapolated total energy in $\mathrm{kJ} \cdot \mathrm{mol}^{-1}$ against inverse number of unit cells in supercell with basis sets of DZ and TZ quality for the systems Ne, Ar, $\mathrm{NH}_{3}, \mathrm{HCN}$.

An important question is for which supercell size the error becomes negligible. A useful magnitude is given by the chemical accuracy of $4 \mathrm{~kJ} \cdot \mathrm{mol}^{-1}$. For weakly-interacting systems such as rare-gas crystals with cohesive energy of less than chemical accuracy, the order of magnitude is set by the cohesive energy itself. As the error of a method should be not larger than chemical accuracy, the allowed error of the supercell method must be at least one order of magnitude smaller then the methodological error, i.e., not larger than $0.4 \mathrm{~kJ} \cdot \mathrm{mol}^{-1}$. We find that a $3 \times 3 \times 3$ super cell provides sufficient accuracy for all functionals and test systems. This behaviour is in agreement with the literature [80]. Sometimes, the total energy per formula unit of the $4 \times 4 \times 4$ super cell has a higher magnitude than this of the $3 \times 3 \times 3$ supercell, which may be due to numerical issues. For PBE, a cubic fit does not seem to be appropriate, and an exponential fit should be used instead.

\subsection{Convergence of the BSSE}

The BSSEs for the different test systems are compiled in Figure 2. First, we would like to point out that the BSSE is significantly larger for the molecular crystals than for the rare-gas crystals. This might be related to the larger number of atoms per molecule and to the spread of the basis functions. Since the effective core charge of rare-gas atoms is larger than for carbon or nitrogen, the basis functions are more localized which results in weaker overlap with neighbouring atoms. This is supported by the 
smaller reduction in BSSE for Ar and Ne when we exploit larger basis sets. Thus, augmentation of basis sets must significantly reduce BSSEs of Ar and Ne. Indeed, diffuse basis functions actually improve cohesive energies as shown by Sansone et al. [63].
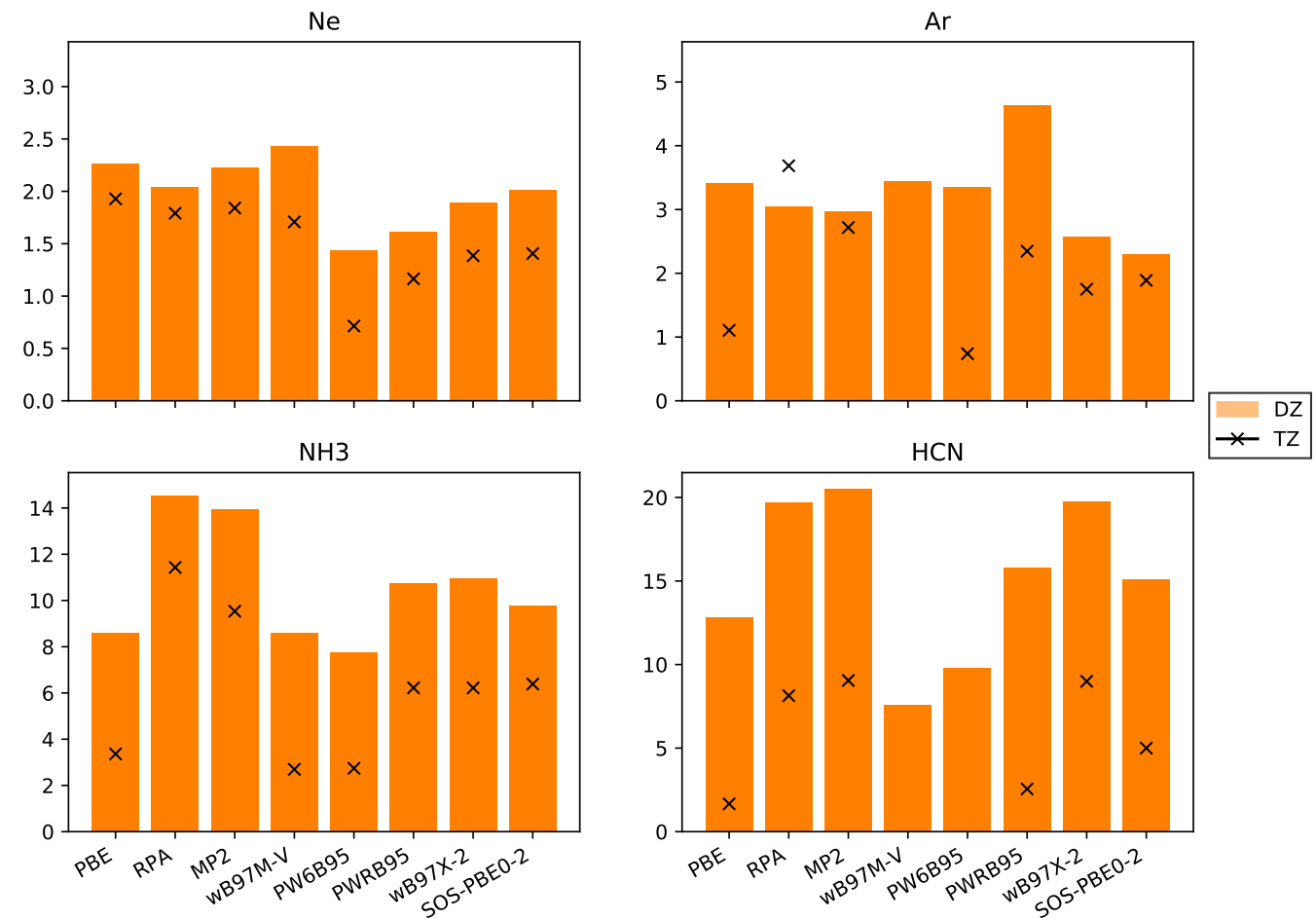

Figure 2. Negative Basis set superposition errors in $\mathrm{kJ} \cdot \mathrm{mol}^{-1}$ with basis sets of $\mathrm{DZ}$ and $\mathrm{TZ}$ quality for the systems $\mathrm{Ne}, \mathrm{Ar}, \mathrm{NH}_{3}, \mathrm{HCN}$.

Molecular crystals are thus more suitable objects to study BSSE than rare-gas crystals. For both molecular crystals in the test set, the non-DHDFs PBE, PW6B95 and $\omega$ B97M-V, provide the smallest BSSEs whereas the two WFC methods MP2 and RPA have the largest BSSEs, as expected. The DHDFs have a BSSE between both classes of methods because they employ a mixture of DFT and WFC methods.

Furthermore, we note that the WFC methods in CP2K are implemented within the RI approximation employing an auxiliary basis set. This leads to an additional source of BSSE for RPA, MP2 and all the DHDFs because the addition of the auxiliary functions of the ghost atoms increases the overall accuracy.

\subsection{Convergence with Respect to Basis Set Size}

In numerous studies, it was shown that total energies from DFT calculations converge exponentially with respect to basis set size. In contrast to that, total energies from WFC methods converge cubically with respect to basis set size when employing correlation-consistent basis sets. Thus, most DHDFs are expected to have a cubic convergence with respect to basis set size but with a smaller prefactor. DHDFs employing a long-ranged Coulomb operator only and describing short-ranged interactions with a density functional, converge exponentially [40]. This behaviour is confirmed with our data compiled in Figure 3. 

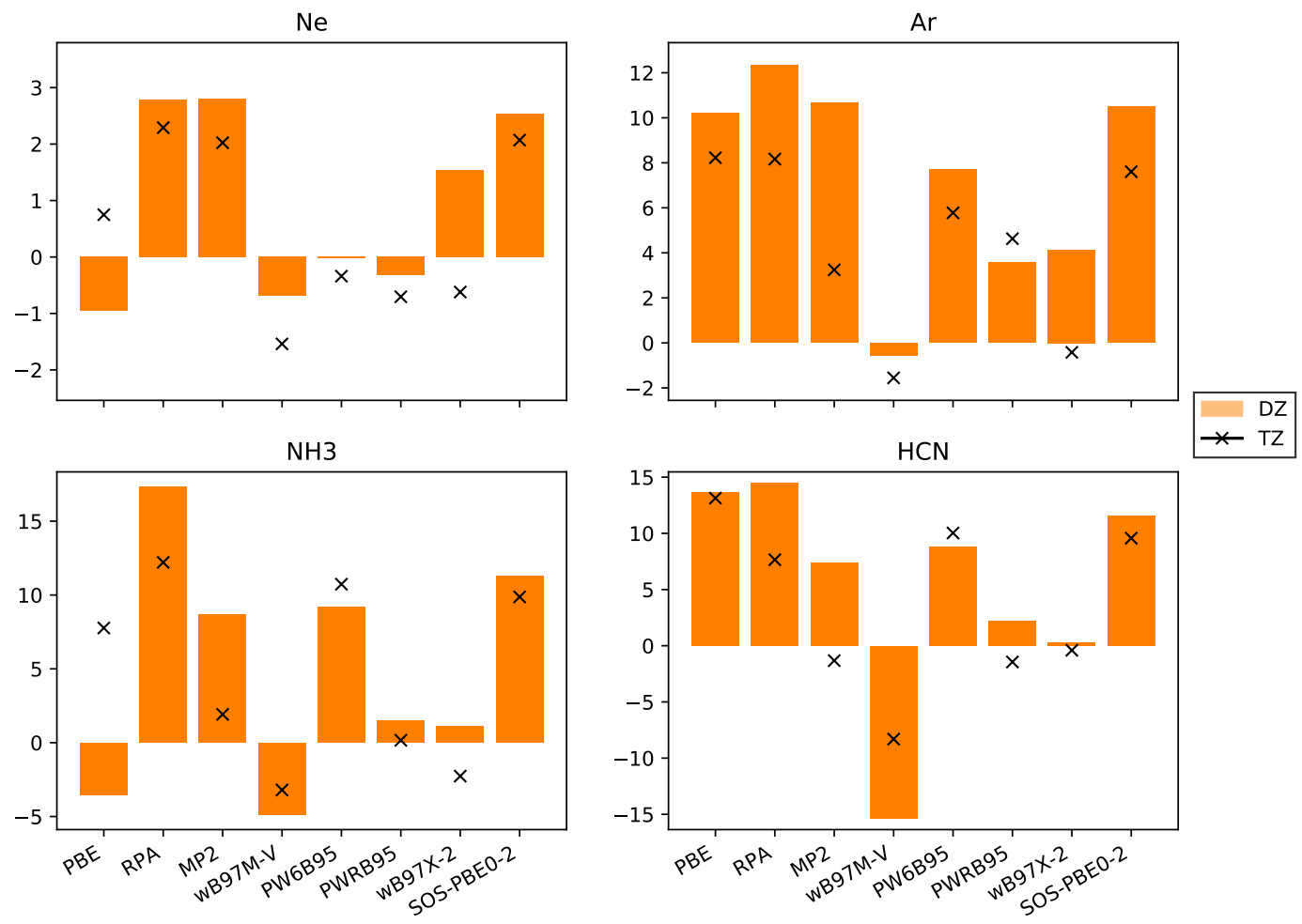

Figure 3. Errors in cohesive energies in $\mathrm{kJ} \cdot \mathrm{mol}^{-1}$ with respect to the experimental values with basis sets of $\mathrm{DZ}$ and $\mathrm{TZ}$ quality for the systems $\mathrm{Ne}, \mathrm{Ar}, \mathrm{NH}_{3}, \mathrm{HCN}$.

Since larger basis sets systematically reduce total energies, cohesive energies increase. We observe this behaviour for the WFC methods and almost all DHDFs. The slight difference for PWRB95 in case of Ar may be due to not full convergence with respect to super cell size. For the other functionals-PBE, $\omega \mathrm{B} 97 \mathrm{M}-\mathrm{V}$ and PW6B95-the cohesive energy from the TZ basis set is sometimes higher, i.e., the system is weaker bound. One problem might be that the $2 \times 2 \times 2$ super cells are not yet fully converged or the extrapolation scheme using a linear fit of the total energies versus the inverse of the volume is not appropriate and an exponential fit might be more suitable.

Next, we would like to discuss the results obtained for the molecular crystals $\mathrm{NH}_{3}$ and $\mathrm{HCN}$. They are bound together by covalent bonds, dipole-dipole interactions, and dispersion interactions. For both systems, the results with the RPA and MP2 methods significantly improve the results over GGA DFT functionals, MP2 even achieving chemical accuracy. The $\omega$ B97M-V functional also provides very accurate numbers. The PW6B95 functional, as PBE, systematically underestimates the cohesive energies with errors compatible to PBE. The PWRB95 functional significantly improves upon the results of its relative PW6B95, bringing them within $1 \mathrm{~kJ} \cdot \mathrm{mol}^{-1}$ from the experiment. The same holds for the $\omega \mathrm{B} 97 \mathrm{X}-2$ functional compared with the $\omega \mathrm{B} 97 \mathrm{M}-\mathrm{V}$ functional, although the DHDF is based on the non-meta-GGA HDF $\omega$ B97X [86]. One of the worst performing functionals is SOS-PBE0-2.

For the rare-gas crystals, the picture is more complicated because the absolute values of the cohesive energies are of the order of the chemical accuracy. As pointed out by Sansone et al. [63], augmented basis sets are required for these systems. Our cohesive energies from MP2 with a TZ basis set are only slightly lower than their result with a DZ basis set but still much worse than those with an augmented basis set for both $\mathrm{Ne}$ and Ar. Consequently, our results for $\mathrm{Ne}$ do not allow for an evaluation of the performance of these functionals and further studies employing either quadruple or augmented basis sets (which are to be constructed) are needed. Nevertheless, our results for Ar 
show that the $\omega$ B97X-2 functional provides a good description. The same holds for MP2, PW6B95 and PWRB95, although one needs further investigations with augmented basis sets.

This issue does not apply to the molecular crystals. Indeed, our cohesive energies with a TZ basis set are even lower than those with an augmented basis set. Thus, the use of augmented basis sets is not necessary for the molecular crystals. This result is important for reducing computational costs of HF calculations and low-scaling WFC methods.

\section{Discussion}

Because DHDFs can be considered to be a mixture of DFT and WFC methods, the flexibility of DHDF parametrizations can yield approaches more accurate than the parent DFT and WFC functionals. At the same time, they inherit the shortcomings of both classes. Due to the dependence on the grid parameters, the functionals PW6B95 and PWRB95 are more difficult to use than others: care must be taken to check whether the results are converged with respect to the grid parameters, in $\mathrm{CP} 2 \mathrm{~K}$, the density cutoff.

As expected, PBE can only provide the order of magnitude for weakly interacting systems, although it converges fast with respect to basis set size and has a low BSSE. MP2 and RPA are more sensitive to the basis set size and exhibit large BSSEs. These methods provide a moderate accuracy for different systems with small basis sets.

Non-DHDFs benefit from lower BSSEs. The PW6B95 functional has high demands on integration grids. Both considered functionals also provide a moderate accuracy and should be favourable over MP2 and RPA with their higher computational costs.

The double-hybrid functionals PWRB95 and $\omega$ B97X-2 show excellent performance with moderate BSSEs and lower basis set incompleteness errors. Both have computational costs compatible to full MP2 or RPA calculations and inherit the need of fine integration grids for accurate results, especially for PWRB95.

The non-empirical SOS-MP2 based DHDF, SOS-PBE0-2, does not provide any advantage as compared to the original methods. It was pointed out by different authors $[87,88]$ that non-empirical DHDFs usually perform worse than empirical DHDFs.

\section{Conclusions}

In this study, we examined a selection of different HDFs and DHDFs by computing cohesive energies in four different crystal structures. Our results show that DHDFs inherit the shortcomings of the underlying DFT functional (integration grids) and the underlying WFC method (computational costs, BSSE, basis set dependence). We were able to show that the PWRB95 and the $\omega$ B97X-2 functionals provide excellent accuracy for molecular and rare-gas crystals. The HDFs $\omega$ B97M-V and PW6B95 also provide reasonable accuracy for these systems, whereas the SOS-PBE0-2 functional underperforms and can not be recommended.

The exploited basis sets allow a good description of molecular crystals. For the rare-gas crystals, we showed that non-augmented basis sets are not sufficient to achieve energy convergence with respect to the basis set size. Due to the high computational costs, we leave studies with augmented basis sets (and the construction of those basis sets) as well as benchmarking more range-separated DHDFs for future prospect.

Author Contributions: Conceptualization, J.H.; methodology, F.S.; software, F.S.; validation, F.S.; formal analysis, F.S.; investigation, F.S.; resources, J.H. and V.V.R.; data curation, F.S.; writing-original draft preparation, F.S. and V.V.R.; writing - review and editing, V.V.R. and J.H.; visualization, F.S.; supervision, J.H. and V.V.R.; project administration, J.H.; funding acquisition, J.H. All authors have read and agreed to the published version of the manuscript.

Funding: This work is supported by the MARVEL National Centre for Competency in Research funded by the Swiss National Science Foundation (grant agreement ID 51NF40-182892). 
Acknowledgments: This work was supported by a grant from the Swiss National Supercomputing Centre (CSCS) under project IDs mr25 and uzh1.

Conflicts of Interest: The authors declare no conflict of interest.

\section{Abbreviations}

The following abbreviations are used in this manuscript:

DFT Density Functional Theory

DHDF Double-Hybrid Density Functional

DZ Double Zeta

GTO Gaussian Type Orbital

HDF Hybrid Density Functional

HF Hartree-Fock

KS Kohn-Sham

MP2 second order Moller-Plesset Perturbation theory

PW Plane Wave

RI Resolution of the Identity

RPA Random-Phase Approximation

SOS-MP2 Scaled Opposite-Spin MP2

TZ Triple Zeta

WFC Wave-Function Correlation

WFT Wave-Function Theory

XC Exchange-Correlation

\section{Appendix A. Pseudopotentials}

Appendix A.1. PBE Pseudopotential

Table A1. PBE pseudopotential parameters. The format corresponds to [78]. Pseudopotentials are taken from [79].

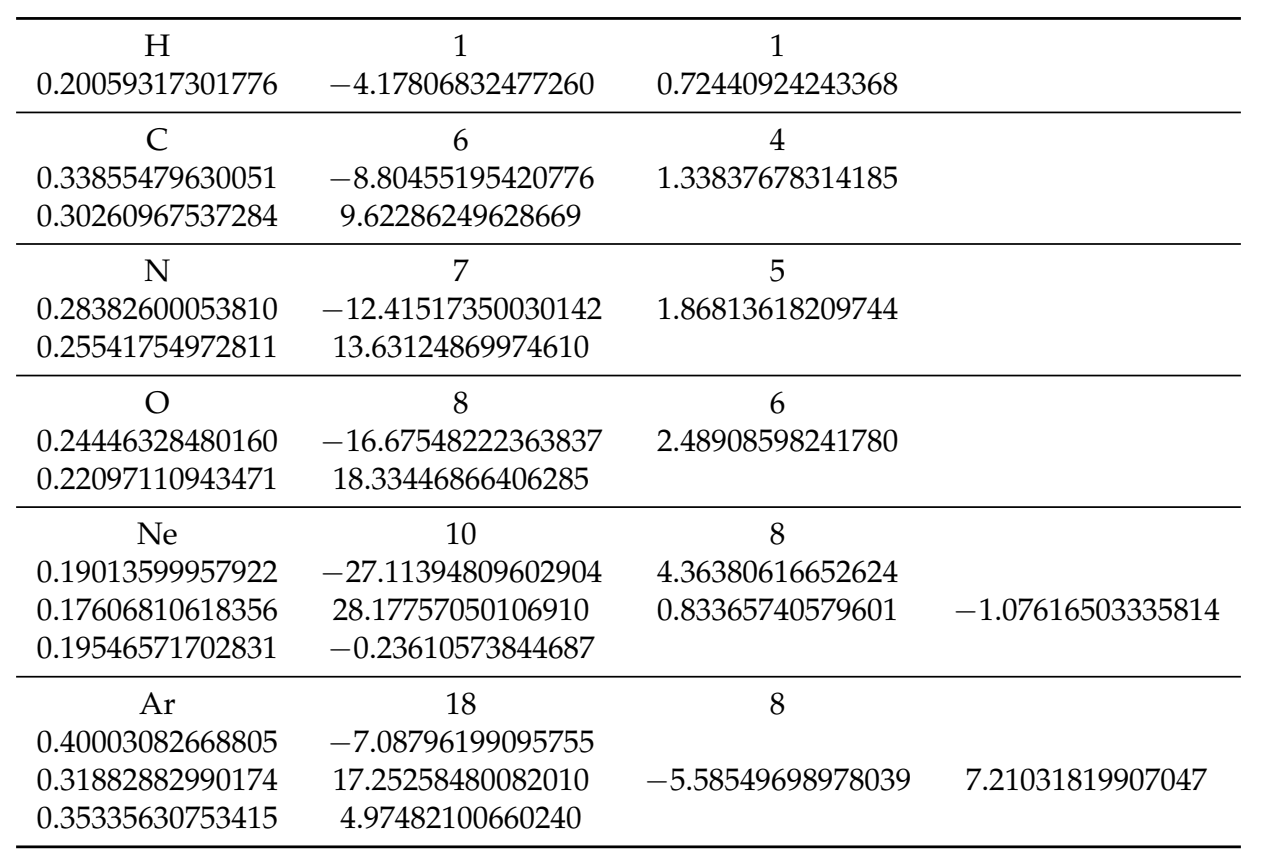


Appendix A.2. PBEO Pseudopotential

Table A2. PBE0 pseudopotential parameters. The format corresponds to [78]. Pseudopotentials are taken from [79].

\begin{tabular}{|c|c|c|c|}
\hline $\begin{array}{c}\mathrm{H} \\
0.20049539759096\end{array}$ & $\begin{array}{c}1 \\
-4.17780338804233\end{array}$ & $\begin{array}{c}1 \\
0.72403926676805\end{array}$ & \\
\hline $\mathrm{C}$ & 6 & 4 & \\
\hline 0.34015230644208 & -8.75626046428525 & 1.33212403341974 & \\
\hline 0.30255799930084 & 9.58980355283555 & & \\
\hline $\mathrm{N}$ & 7 & 5 & \\
\hline 0.28405138134082 & -12.39652421586226 & 1.86372383417056 & \\
\hline 0.25538070446385 & 13.63073438324169 & & \\
\hline $\mathrm{O}$ & 8 & 6 & \\
\hline 0.24671011902360 & -16.65533253748591 & 2.50854752353111 & \\
\hline 0.22100154713718 & 18.34370453997725 & & \\
\hline $\mathrm{Ne}$ & 10 & 8 & \\
\hline 0.19050423878827 & -27.40404160755363 & 4.42644219542327 & 0.00312528175949 \\
\hline 0.17609378094694 & 28.18364816845336 & 0.83365182689679 & -1.06378213860369 \\
\hline 0.19427680906964 & -0.23683812086750 & & \\
\hline $\mathrm{Ar}$ & 18 & 8 & \\
\hline 0.39979462541098 & -7.23417721420866 & 0.00616780402846 & \\
\hline 0.31880599007091 & 17.21513221796928 & -5.58548607072547 & 7.21072495408957 \\
\hline 0.35343634803616 & 4.97384170460103 & & \\
\hline
\end{tabular}

Appendix A.3. HF Pseudopotential

Table A3. Hartree-Fock pseudopotential parameters. The format corresponds to [78]. Pseudopotentials are taken from [79].

\begin{tabular}{cccc}
\hline $\mathrm{H}$ & 1 & 1 & \\
0.20049539759096 & -4.17780338804233 & 0.72403926676805 & \\
\hline $\mathrm{C}$ & 6 & 4 & \\
0.34816792458406 & -8.54312820557867 & 1.33276540541946 & \\
0.30230247000627 & 9.59710582360109 & & \\
\hline $\mathrm{N}$ & 7 & & \\
0.28300476743411 & -12.39840200798251 & 1.86939057420079 & \\
0.25539202567537 & 13.64483766978610 & & \\
\hline $\mathrm{O}$ & 8 & & \\
0.24676969870316 & -16.66528269564613 & 2.52030687064467 & \\
0.22121058101998 & 18.39425181647437 & & \\
\hline $\mathrm{Ne}$ & 10 & & \\
0.19050265092574 & -27.39590696172339 & 4.41958869715540 & 0.01834396326683 \\
0.17637388496062 & 28.18533818441574 & 0.83365996989179 & -1.04842942962620 \\
0.19585379054851 & -0.27609661906079 & & \\
\hline $\mathrm{Ar}$ & 18 & & \\
0.39771927261258 & -7.21348927487361 & 0.01323122557817 & \\
0.31872450490949 & 17.20921819285275 & -5.58549109340678 & 7.19978913165534 \\
0.35357441343299 & 4.98951929408379 & & \\
\hline
\end{tabular}




\section{Appendix B. Primary Basis Sets}

Table A4. Parameters of the cc-DZVP basis set of Hydrogen. Parameters taken from [80].

\begin{tabular}{ccc}
\hline Shell Type & Exponents & Contraction Coefficients \\
\hline $\mathrm{s}$ & 8.3744350009 & -0.0283380461 \\
& 1.8058681460 & -0.1333810052 \\
& 0.4852528328 & -0.3995676063 \\
$\mathrm{~s}$ & 0.1658236932 & 1.0000000000 \\
$\mathrm{p}$ & 0.7270000000 & 1.0000000000 \\
\hline
\end{tabular}

Table A5. Parameters of the cc-TZVP basis set of Hydrogen. Parameters taken from [80].

\begin{tabular}{ccc}
\hline Shell Type & Exponents & Contraction Coefficients \\
\hline $\mathrm{s}$ & 10.8827241585 & -0.0167058885 \\
& 3.0968750876 & -0.0627538300 \\
& 0.9874518162 & -0.1917521975 \\
$\mathrm{~s}$ & 0.3450687533 & 1.0000000000 \\
$\mathrm{~s}$ & 0.1492693554 & 1.0000000000 \\
$\mathrm{p}$ & 1.4070000000 & 1.0000000000 \\
$\mathrm{p}$ & 0.3880000000 & 1.0000000000 \\
$\mathrm{~d}$ & 1.0570000000 & 1.0000000000 \\
\hline
\end{tabular}

Table A6. Parameters of the cc-DZVP basis set of Carbon. Parameters taken from [80].

\begin{tabular}{cccc}
\hline Shell Type & Exponents & \multicolumn{2}{c}{ Contraction Coefficients } \\
\hline $\mathrm{sp}$ & 4.3362376436 & 0.1490797872 & -0.0878123619 \\
& 1.2881838513 & -0.0292640031 & -0.2775560300 \\
& 0.4037767149 & -0.6882040510 & -0.4712295093 \\
$\mathrm{sp}$ & 0.1187877657 & 1.0000000000 & 1.0000000000 \\
$\mathrm{~d}$ & 0.5500000000 & 1.0000000000 & \\
\hline
\end{tabular}

Table A7. Parameters of the cc-TZVP basis set of Carbon. Parameters taken from [80].

\begin{tabular}{cccc}
\hline Shell Type & Exponents & \multicolumn{2}{c}{ Contraction Coefficients } \\
\hline $\mathrm{sp}$ & 5.3685662937 & 0.0974901974 & -0.0510969367 \\
& 1.9830691554 & 0.1041996677 & -0.1693035193 \\
& 0.6978346167 & -0.3645093878 & -0.3579933930 \\
$\mathrm{sp}$ & 0.2430968816 & 1.0000000000 & 1.0000000000 \\
$\mathrm{sp}$ & 0.0812865018 & 1.0000000000 & 1.0000000000 \\
$\mathrm{~d}$ & 1.0970000000 & 1.0000000000 & \\
$\mathrm{~d}$ & 0.3180000000 & 1.0000000000 & \\
$\mathrm{f}$ & 0.7610000000 & 1.0000000000 & \\
\hline
\end{tabular}

Table A8. Parameters of the cc-DZVP basis set of Nitrogen. Parameters taken from [80].

\begin{tabular}{cccc}
\hline Shell Type & Exponents & \multicolumn{2}{c}{ Contraction Coefficients } \\
\hline $\mathrm{sp}$ & 6.1526903413 & 0.1506300537 & -0.0950603476 \\
& 1.8236332280 & -0.0360100734 & -0.2918864295 \\
& 0.5676628870 & -0.6942023212 & -0.4739050050 \\
$\mathrm{sp}$ & 0.1628222852 & 1.0000000000 & 1.0000000000 \\
$\mathrm{~d}$ & 0.8170000000 & 1.0000000000 & \\
\hline
\end{tabular}


Table A9. Parameters of the cc-TZVP basis set of Nitrogen. Parameters taken from [80].

\begin{tabular}{cccc}
\hline Shell Type & Exponents & \multicolumn{2}{c}{ Contraction Coefficients } \\
\hline $\mathrm{sp}$ & 7.6227447102 & 0.0983924689 & -0.0561654555 \\
& 2.7970605447 & 0.1045217098 & -0.1798165209 \\
& 0.9909765447 & -0.3742661352 & -0.3653986185 \\
$\mathrm{sp}$ & 0.3417314862 & 1.0000000000 & 1.0000000000 \\
$\mathrm{sp}$ & 0.1116822743 & 1.0000000000 & 1.0000000000 \\
$\mathrm{~d}$ & 1.6540000000 & 1.0000000000 & \\
$\mathrm{~d}$ & 0.4690000000 & 1.0000000000 & \\
$\mathrm{f}$ & 1.0930000000 & 1.0000000000 & \\
\hline
\end{tabular}

Table A10. Parameters of the cc-DZVP basis set of Oxygen. Parameters taken from [80].

\begin{tabular}{cccc}
\hline Shell Type & Exponents & \multicolumn{2}{c}{ Contraction Coefficients } \\
\hline $\mathrm{sp}$ & 8.3043855492 & 0.1510165999 & -0.0995679273 \\
& 2.4579484191 & -0.0393195364 & -0.3011422449 \\
& 0.7597373434 & -0.6971724029 & -0.4750857083 \\
$\mathrm{sp}$ & 0.2136388632 & 1.0000000000 & 1.0000000000 \\
$\mathrm{~d}$ & 1.1850000000 & 1.0000000000 & \\
\hline
\end{tabular}

Table A11. Parameters of the cc-TZVP basis set of Oxygen. Parameters taken from [80].

\begin{tabular}{cccc}
\hline Shell Type & Exponents & \multicolumn{2}{c}{ Contraction Coefficients } \\
\hline $\mathrm{sp}$ & 10.2674419938 & 0.0989598460 & -0.0595856940 \\
& 3.7480495696 & 0.1041178339 & -0.1875649045 \\
& 1.3308337704 & -0.3808255700 & -0.3700707718 \\
$\mathrm{sp}$ & 0.4556802254 & 1.0000000000 & 1.0000000000 \\
$\mathrm{sp}$ & 0.1462920596 & 1.0000000000 & 1.0000000000 \\
$\mathrm{~d}$ & 2.3140000000 & 1.0000000000 & \\
$\mathrm{~d}$ & 0.6450000000 & 1.0000000000 & \\
$\mathrm{f}$ & 1.4280000000 & 1.0000000000 & \\
\hline
\end{tabular}

Table A12. Parameters of the cc-DZVP basis set of Neon.

\begin{tabular}{cccc}
\hline Shell Type & Exponents & \multicolumn{2}{c}{ Contraction Coefficients } \\
\hline $\mathrm{sp}$ & 13.8523672900 & 0.1501498200 & 0.10214300 \\
& 4.0685498000 & -0.0314908700 & 0.3058092400 \\
& 1.2730584300 & -0.7070497300 & 0.4766050400 \\
$\mathrm{sp}$ & 0.3565013600 & 1.0000000000 & 1.0000000000 \\
$\mathrm{~d}$ & 2.2020000000 & 1.0000000000 & \\
\hline
\end{tabular}

Table A13. Parameters of the cc-TZVP basis set of Neon.

\begin{tabular}{cccc}
\hline Shell Type & Exponents & \multicolumn{2}{c}{ Contraction Coefficients } \\
\hline $\mathrm{sp}$ & 17.4276488400 & 0.073686700 & 0.0702714400 \\
& 6.3439264100 & 0.0969132500 & 0.2167849700 \\
$\mathrm{sp}$ & 2.2823205800 & -0.3010470300 & 0.4317763300 \\
$\mathrm{sp}$ & 0.7945993700 & 1.0000000000 & 1.0000000000 \\
$\mathrm{~d}$ & 4.2560537300 & 1.0000000000 & 1.0000000000 \\
$\mathrm{~d}$ & 1.0960000000 & 1.0000000000 & \\
$\mathrm{f}$ & 2.5440000000 & 1.0000000000 & \\
\hline
\end{tabular}


Table A14. Parameters of the cc-DZVP basis set of Argon.

\begin{tabular}{cccc}
\hline Shell Type & Exponents & \multicolumn{2}{c}{ Contraction Coefficients } \\
\hline $\mathrm{sp}$ & 2.6724631600 & 0.1547491900 & 0.2663267700 \\
& 1.5750569800 & -0.1300613000 & -1.0821938600 \\
& 0.5528926600 & -0.1247859600 & 0.1177549000 \\
$\mathrm{sp}$ & 0.1720724500 & 1.0000000000 & 1.0000000000 \\
$\mathrm{~d}$ & 0.7380000000 & 1.0000000000 & \\
\hline
\end{tabular}

Table A15. Parameters of the cc-TZVP basis set of Argon.

\begin{tabular}{cccc}
\hline Shell Type & Exponents & \multicolumn{2}{c}{ Contraction Coefficients } \\
\hline $\mathrm{sp}$ & 3.5650652500 & -0.03560400 & -0.0341601300 \\
& 2.8711385000 & 0.107453000 & 0.0274003200 \\
& 0.928908200 & -0.070935800 & 0.1084604500 \\
$\mathrm{sp}$ & 0.3762992800 & 1.0000000000 & 1.0000000000 \\
$\mathrm{sp}$ & 0.1388133000 & 1.0000000000 & 1.0000000000 \\
$\mathrm{~d}$ & 1.2540000000 & 1.0000000000 & \\
$\mathrm{~d}$ & 0.4100000000 & 1.0000000000 & \\
$\mathrm{f}$ & 0.8900000000 & 1.0000000000 & \\
\hline
\end{tabular}

\section{Appendix C. Auxiliary Basis Sets}

Table A16. Parameters of the cc-DZVP auxiliary basis set of Hydrogen. Parameters taken from [89].

\begin{tabular}{ccc}
\hline Shell Type & Exponents & Contraction Coefficients \\
\hline $\mathrm{s}$ & 5.1153315245 & 1.0000000000 \\
$\mathrm{~s}$ & 1.1472440266 & 1.0000000000 \\
$\mathrm{~s}$ & 0.3203181150 & 1.0000000000 \\
$\mathrm{p}$ & 1.9149400132 & 1.0000000000 \\
$\mathrm{p}$ & 0.9859513111 & 1.0000000000 \\
$\mathrm{~d}$ & 1.1714848284 & 1.0000000000 \\
\hline
\end{tabular}

Table A17. Parameters of the cc-TZVP auxiliary basis set of Hydrogen. Parameters taken from [89].

\begin{tabular}{ccc}
\hline Shell Type & Exponents & Contraction Coefficients \\
\hline s & 8.5115919487 & 1.0000000000 \\
s & 1.8744684087 & 1.0000000000 \\
s & 0.5632515602 & 1.0000000000 \\
s & 0.3698299759 & 1.0000000000 \\
p & 2.3711712242 & 1.0000000000 \\
p & 1.1794161391 & 1.0000000000 \\
p & 0.6050431621 & 1.0000000000 \\
d & 1.8092525711 & 1.0000000000 \\
d & 1.1433220615 & 1.0000000000 \\
f & 1.8065804513 & 1.0000000000 \\
\hline
\end{tabular}


Table A18. Parameters of the cc-DZVP auxiliary basis set of Carbon. Parameters taken from [89].

\begin{tabular}{ccc}
\hline Shell Type & Exponents & Contraction Coefficients \\
\hline S & 13.8045000000 & 1.0000000000 \\
S & 4.7727700000 & 1.0000000000 \\
S & 1.5133300005 & 1.0000000000 \\
S & 0.7826969986 & 1.0000000000 \\
S & 0.4090720022 & 1.0000000000 \\
S & 0.2067960415 & 1.0000000000 \\
p & 6.0052300018 & 1.0000000000 \\
p & 1.7206000311 & 1.0000000000 \\
p & 0.7544648237 & 1.0000000000 \\
p & 0.3216662007 & 1.0000000000 \\
d & 2.6784400611 & 1.0000000000 \\
d & 0.9225147829 & 1.0000000000 \\
d & 0.3408412315 & 1.0000000000 \\
f & 2.7429299969 & 1.0000000000 \\
f & 0.8957560323 & 1.0000000000 \\
\hline
\end{tabular}

Table A19. Parameters of the cc-TZVP auxiliary basis set of Carbon. Parameters taken from [89].

\begin{tabular}{ccc}
\hline Shell Type & Exponents & Contraction Coefficients \\
\hline s & 22.2608165950 & 1.0000000000 \\
s & 7.1315246807 & 1.0000000000 \\
s & 3.5380450775 & 1.0000000000 \\
s & 1.2333453175 & 1.0000000000 \\
s & 0.2821517353 & 1.0000000000 \\
s & 0.3468258230 & 1.0000000000 \\
p & 7.2975063903 & 1.0000000000 \\
p & 3.7896065213 & 1.0000000000 \\
p & 1.0633834831 & 1.0000000000 \\
p & 0.2356430320 & 1.0000000000 \\
p & 0.5078423493 & 1.0000000000 \\
d & 9.9000557486 & 1.0000000000 \\
d & 2.3408375066 & 1.0000000000 \\
d & 1.5195338451 & 1.0000000000 \\
d & 0.5788522388 & 1.0000000000 \\
d & 0.3721345858 & 1.0000000000 \\
f & 1.9332589728 & 1.0000000000 \\
f & 1.1560553410 & 1.0000000000 \\
f & 0.4987261239 & 1.0000000000 \\
g & 1.2175667359 & 1.0000000000 \\
\hline
\end{tabular}

Table A20. Parameters of the cc-DZVP auxiliary basis set of Nitrogen. Parameters taken from [89].

\begin{tabular}{ccc}
\hline Shell Type & Exponents & Contraction Coefficients \\
\hline s & 20.4678978643 & 1.0000000000 \\
s & 7.6243888531 & 1.0000000000 \\
s & 2.3446722210 & 1.0000000000 \\
s & 1.1234062160 & 1.0000000000 \\
s & 0.7258555682 & 1.0000000000 \\
s & 0.3516451521 & 1.0000000000 \\
p & 8.5530798511 & 1.0000000000 \\
p & 2.5349440268 & 1.0000000000 \\
p & 1.0857134625 & 1.0000000000 \\
p & 0.4193736786 & 1.0000000000 \\
d & 3.4384121802 & 1.0000000000 \\
d & 1.2761051199 & 1.0000000000 \\
d & 0.3971760294 & 1.0000000000 \\
f & 3.4010871209 & 1.0000000000 \\
f & 1.3350506486 & 1.0000000000 \\
\hline
\end{tabular}


Table A21. Parameters of the cc-TZVP auxiliary basis set of Nitrogen. Parameters taken from [89].

\begin{tabular}{ccc}
\hline Shell Type & Exponents & Contraction Coefficients \\
\hline $\mathrm{s}$ & 21.6812818892 & 1.0000000000 \\
$\mathrm{~s}$ & 7.9027065688 & 1.0000000000 \\
$\mathrm{~s}$ & 2.4447520737 & 1.0000000000 \\
$\mathrm{~s}$ & 1.2617706294 & 1.0000000000 \\
$\mathrm{~s}$ & 0.8067419821 & 1.0000000000 \\
$\mathrm{~s}$ & 0.2885279906 & 1.0000000000 \\
$\mathrm{p}$ & 10.3296673020 & 1.0000000000 \\
$\mathrm{p}$ & 2.9182107455 & 1.0000000000 \\
$\mathrm{p}$ & 1.3834177164 & 1.0000000000 \\
$\mathrm{p}$ & 0.7162830530 & 1.0000000000 \\
$\mathrm{p}$ & 0.3296257918 & 1.0000000000 \\
$\mathrm{~d}$ & 13.9094333585 & 1.0000000000 \\
$\mathrm{~d}$ & 4.5822351942 & 1.0000000000 \\
$\mathrm{~d}$ & 2.1943496520 & 1.0000000000 \\
$\mathrm{~d}$ & 0.8349245145 & 1.0000000000 \\
$\mathrm{~d}$ & 0.4510857395 & 1.0000000000 \\
$\mathrm{f}$ & 3.4744937308 & 1.0000000000 \\
$\mathrm{f}$ & 1.5532348673 & 1.0000000000 \\
$\mathrm{f}$ & 0.8522508678 & 1.0000000000 \\
$\mathrm{~g}$ & 1.7674440596 & 1.0000000000 \\
\hline
\end{tabular}

Table A22. Parameters of the cc-DZVP auxiliary basis set of Oxygen. Parameters taken from [89].

\begin{tabular}{ccc}
\hline Shell Type & Exponents & Contraction Coefficients \\
\hline S & 25.5779913844 & 1.0000000000 \\
S & 9.5515670675 & 1.0000000000 \\
s & 2.9409752222 & 1.0000000000 \\
S & 1.3964896911 & 1.0000000000 \\
S & 0.9105756313 & 1.0000000000 \\
s & 0.4821009543 & 1.0000000000 \\
p & 10.8823093197 & 1.0000000000 \\
p & 3.2132775587 & 1.0000000000 \\
p & 1.3802086101 & 1.0000000000 \\
p & 0.4601246170 & 1.0000000000 \\
d & 4.5934895346 & 1.0000000000 \\
d & 1.7871052175 & 1.0000000000 \\
d & 0.4206288858 & 1.0000000000 \\
f & 4.2218855419 & 1.0000000000 \\
f & 1.7894864633 & 1.0000000000 \\
\hline
\end{tabular}


Table A23. Parameters of the cc-TZVP auxiliary basis set of Oxygen. Parameters taken from [89].

\begin{tabular}{ccc}
\hline Shell Type & Exponents & Contraction Coefficients \\
\hline $\mathrm{s}$ & 24.5595006061 & 1.0000000000 \\
$\mathrm{~s}$ & 8.3254503805 & 1.0000000000 \\
$\mathrm{~s}$ & 2.8895585562 & 1.0000000000 \\
$\mathrm{~s}$ & 1.3383587201 & 1.0000000000 \\
$\mathrm{~s}$ & 0.8797495165 & 1.0000000000 \\
$\mathrm{~s}$ & 0.2902204697 & 1.0000000000 \\
$\mathrm{p}$ & 15.0341204959 & 1.0000000000 \\
$\mathrm{p}$ & 3.9838033442 & 1.0000000000 \\
$\mathrm{p}$ & 2.2151496463 & 1.0000000000 \\
$\mathrm{p}$ & 0.8979637674 & 1.0000000000 \\
$\mathrm{p}$ & 0.4128471304 & 1.0000000000 \\
$\mathrm{~d}$ & 15.8683289847 & 1.0000000000 \\
$\mathrm{~d}$ & 5.3913486662 & 1.0000000000 \\
$\mathrm{~d}$ & 2.5385447175 & 1.0000000000 \\
$\mathrm{~d}$ & 1.0911199995 & 1.0000000000 \\
$\mathrm{~d}$ & 0.3766843343 & 1.0000000000 \\
$\mathrm{f}$ & 4.6812603411 & 1.0000000000 \\
$\mathrm{f}$ & 2.1656106741 & 1.0000000000 \\
$\mathrm{f}$ & 1.0331835741 & 1.0000000000 \\
$\mathrm{~g}$ & 2.3079719899 & 1.0000000000 \\
\hline
\end{tabular}

Table A24. Parameters of the cc-DZVP auxiliary basis set of Neon.

\begin{tabular}{ccc}
\hline Shell Type & Exponents & Contraction Coefficients \\
\hline $\mathrm{s}$ & 0.4283083846 & 1.0000000000 \\
$\mathrm{~s}$ & 0.9127866030 & 1.0000000000 \\
$\mathrm{~s}$ & 1.4054659820 & 1.0000000000 \\
$\mathrm{~s}$ & 2.7308004917 & 1.0000000000 \\
$\mathrm{~s}$ & 10.8821914790 & 1.0000000000 \\
$\mathrm{~s}$ & 27.4563627600 & 1.0000000000 \\
$\mathrm{p}$ & 0.7929884909 & 1.0000000000 \\
$\mathrm{p}$ & 2.5008596627 & 1.0000000000 \\
$\mathrm{p}$ & 5.9151957867 & 1.0000000000 \\
$\mathrm{p}$ & 15.3673109753 & 1.0000000000 \\
$\mathrm{~d}$ & 0.8283650682 & 1.0000000000 \\
$\mathrm{~d}$ & 3.3870444721 & 1.0000000000 \\
$\mathrm{~d}$ & 10.9778901482 & 1.0000000000 \\
$\mathrm{f}$ & 3.1170471491 & 1.0000000000 \\
$\mathrm{f}$ & 6.8656878672 & 1.0000000000 \\
\hline
\end{tabular}

Table A25. Parameters of the cc-TZVP auxiliary basis set of Neon.

\begin{tabular}{ccc}
\hline Shell Type & Exponents & Contraction Coefficients \\
\hline s & 0.4218054070 & 1.0000000000 \\
S & 0.9220306716 & 1.0000000000 \\
s & 1.8281445142 & 1.0000000000 \\
s & 4.0122843245 & 1.0000000000 \\
s & 9.3557953735 & 1.0000000000 \\
s & 21.2448698799 & 1.0000000000 \\
p & 0.7819113369 & 1.0000000000 \\
p & 1.2774995450 & 1.0000000000 \\
p & 4.0929391223 & 1.0000000000 \\
p & 6.2303237413 & 1.0000000000 \\
p & 16.9114662817 & 1.0000000000 \\
d & 0.5593906817 & 1.0000000000 \\
d & 1.1997449519 & 1.0000000000 \\
d & 2.6389336208 & 1.0000000000 \\
d & 4.9434479195 & 1.0000000000 \\
d & 14.1003353302 & 1.0000000000 \\
f & 1.5593705359 & 1.0000000000 \\
f & 3.3654768720 & 1.0000000000 \\
f & 8.0058977735 & 1.0000000000 \\
g & 3.9789077704 & 1.0000000000 \\
\hline & &
\end{tabular}


Table A26. Parameters of the cc-DZVP auxiliary basis set of Argon.

\begin{tabular}{ccc}
\hline Shell Type & Exponents & Contraction Coefficients \\
\hline s & 0.2021934524 & 1.0000000000 \\
s & 0.5951644570 & 1.0000000000 \\
S & 0.9713367515 & 1.0000000000 \\
S & 2.1348414404 & 1.0000000000 \\
S & 8.3055209987 & 1.0000000000 \\
s & 24.4838599910 & 1.0000000000 \\
p & 0.3806171008 & 1.0000000000 \\
p & 1.0795771281 & 1.0000000000 \\
p & 1.5490430664 & 1.0000000000 \\
p & 4.5409363372 & 1.0000000000 \\
d & 0.4407961817 & 1.0000000000 \\
d & 1.2550515264 & 1.0000000000 \\
d & 5.8159089208 & 1.0000000000 \\
f & 1.2170432674 & 1.0000000000 \\
f & 9.1164484253 & 1.0000000000 \\
\hline
\end{tabular}

Table A27. Parameters of the cc-TZVP auxiliary basis set of Argon.

\begin{tabular}{ccc}
\hline Shell Type & Exponents & Contraction Coefficients \\
\hline $\mathrm{s}$ & 0.2374198920 & 1.0000000000 \\
$\mathrm{~s}$ & 0.4593140129 & 1.0000000000 \\
$\mathrm{~s}$ & 0.8759707445 & 1.0000000000 \\
$\mathrm{~s}$ & 1.6509598569 & 1.0000000000 \\
$\mathrm{~s}$ & 3.0758784521 & 1.0000000000 \\
$\mathrm{~s}$ & 5.6934758267 & 1.0000000000 \\
$\mathrm{p}$ & 0.2795695538 & 1.0000000000 \\
$\mathrm{p}$ & 0.5599111995 & 1.0000000000 \\
$\mathrm{p}$ & 1.1757695117 & 1.0000000000 \\
$\mathrm{p}$ & 2.6036947304 & 1.0000000000 \\
$\mathrm{p}$ & 5.6658077981 & 1.0000000000 \\
$\mathrm{~d}$ & 0.2898716779 & 1.0000000000 \\
$\mathrm{~d}$ & 0.6972610882 & 1.0000000000 \\
$\mathrm{~d}$ & 1.2166500341 & 1.0000000000 \\
$\mathrm{~d}$ & 2.4487532956 & 1.0000000000 \\
$\mathrm{~d}$ & 5.4801182100 & 1.0000000000 \\
$\mathrm{f}$ & 0.6819926505 & 1.0000000000 \\
$\mathrm{f}$ & 1.4331910682 & 1.0000000000 \\
$\mathrm{f}$ & 3.3654529504 & 1.0000000000 \\
$\mathrm{~g}$ & 1.4464903837 & 1.0000000000 \\
\hline
\end{tabular}

\section{References}

1. Kohn, W.; Sham, L.J. Self-Consistent Equations Including Exchange and Correlation Effects. Phys. Rev. 1965, 140, A1133-A1138. [CrossRef]

2. Hohenberg, P.; Kohn, W. Inhomogeneous Electron Gas. Phys. Rev. 1964, 136, B864-B871. [CrossRef]

3. Perdew, J.P.; Schmidt, K.; Van Doren, V.; Van Alsenoy, C.; Geerlings, P. Jacob's ladder of density functional approximations for the exchange-correlation energy. AIP Conf. Proc. 2001, 577, 1-20. [CrossRef]

4. Slater, J.C. A Simplification of the Hartree-Fock Method. Phys. Rev. 1951, 81, 385-390. [CrossRef]

5. Gell-Mann, M.; Brueckner, K.A. Correlation Energy of an Electron Gas at High Density. Phys. Rev. 1957, 106, 364-368. [CrossRef]

6. Perdew, J.P.; Wang, Y. Accurate and simple analytic representation of the electron-gas correlation energy. Phys. Rev. B 1992, 45, 13244-13249. [CrossRef] [PubMed]

7. Ceperley, D.M.; Alder, B.J. Ground State of the Electron Gas by a Stochastic Method. Phys. Rev. Lett. 1980, 45, 566-569. [CrossRef]

8. Vosko, S.H.; Wilk, L.; Nusair, M. Accurate spin-dependent electron liquid correlation energies for local spin density calculations: A critical analysis. Can. J. Phys. 1980, 58, 1200-1211. [CrossRef] 
9. Lee, C.; Yang, W.; Parr, R.G. Development of the Colle-Salvetti correlation-energy formula into a functional of the electron density. Phys. Rev. B 1988, 37, 785-789. [CrossRef] [PubMed]

10. Perdew, J.P.; Burke, K.; Ernzerhof, M. Generalized Gradient Approximation Made Simple. Phys. Rev. Lett. 1996, 77, 3865-3868. [CrossRef] [PubMed]

11. Kurth, S.; Perdew, J.P.; Blaha, P. Molecular and solid-state tests of density functional approximations: LSD, GGAs, and meta-GGAs. Int. J. Quantum Chem. 1999, 75, 889-909. [CrossRef]

12. Perdew, J.P.; Kurth, S.; Zupan, A.; Blaha, P. Accurate Density Functional with Correct Formal Properties: A Step Beyond the Generalized Gradient Approximation. Phys. Rev. Lett. 1999, 82, 2544-2547. [CrossRef]

13. Van Voorhis, T.; Scuseria, G.E. A novel form for the exchange-correlation energy functional. J. Chem. Phys. 1998, 109, 400-410. [CrossRef]

14. Tao, J.; Perdew, J.P.; Staroverov, V.N.; Scuseria, G.E. Climbing the Density Functional Ladder: Nonempirical Meta-Generalized Gradient Approximation Designed for Molecules and Solids. Phys. Rev. Lett. 2003, 91, 146401. [CrossRef] [PubMed]

15. Adamo, C.; Barone, V. Toward reliable density functional methods without adjustable parameters: The PBE0 model. J. Chem. Phys. 1999, 110, 6158-6170. [CrossRef]

16. Becke, A.D. Density-functional thermochemistry. III. The role of exact exchange. J. Chem. Phys. 1993, 98, 5648-5652. [CrossRef]

17. Hartree, D.R. The Wave Mechanics of an Atom with a Non-Coulomb Central Field. Part II. Some Results and Discussion. Math. Proc. Camb. Philos. Soc. 1928, 24, 111-132. [CrossRef]

18. Fock, V. Näherungsmethode zur Lösung des quantenmechanischen Mehrkörperproblems. Z. Phys. 1930, 61, 126-148. [CrossRef]

19. Heyd, J.; Scuseria, G.E.; Ernzerhof, M. Hybrid functionals based on a screened Coulomb potential. J. Chem. Phys. 2003, 118, 8207-8215. [CrossRef]

20. Mardirossian, N.; Head-Gordon, M. $\omega$ B97X-V: A 10-parameter, range-separated hybrid, generalized gradient approximation density functional with nonlocal correlation, designed by a survival-of-the-fittest strategy. Phys. Chem. Chem. Phys. 2014, 16, 9904-9924. [CrossRef]

21. Grimme, S. Semiempirical hybrid density functional with perturbative second-order correlation. J. Chem. Phys. 2006, 124, 034108. [CrossRef]

22. Görling, A.; Levy, M. Exact Kohn-Sham scheme based on perturbation theory. Phys. Rev. A 1994, 50, $196-204$. [CrossRef]

23. Zhang, I.Y.; Xu, X. Doubly hybrid density functional for accurate description of thermochemistry, thermochemical kinetics and nonbonded interactions. Int. Rev. Phys. Chem. 2011, 30, 115-160. [CrossRef]

24. Goerigk, L.; Grimme, S. Double-hybrid density functionals. WIREs Comput. Mol. Sci. 2014, 4, 576-600. [CrossRef]

25. Brémond, E.; Ciofini, I.; Sancho-García, J.C.; Adamo, C. Nonempirical Double-Hybrid Functionals: An Effective Tool for Chemists. Accounts Chem. Res. 2016, 49, 1503-1513, [CrossRef] [PubMed]

26. Martin, J.M.L.; Santra, G. Empirical Double-Hybrid Density Functional Theory: A 'Third Way' in Between WFT and DFT. Isr. J. Chem. 2020, 60, 787-804. [CrossRef]

27. Elstner, M.; Hobza, P.; Frauenheim, T.; Suhai, S.; Kaxiras, E. Hydrogen bonding and stacking interactions of nucleic acid base pairs: A density-functional-theory based treatment. J. Chem. Phys. 2001, 114, 5149-5155. [CrossRef]

28. Grimme, S. Accurate description of van der Waals complexes by density functional theory including empirical corrections. J. Comput. Chem. 2004, 25, 1463-1473. [CrossRef]

29. Jurecka, P.; Cerny, J.; Hobza, P.; Salahub, D.R. Density functional theory augmented with an empirical dispersion term. Interaction energies and geometries of 80 noncovalent complexes compared with ab initio quantum mechanics calculations. J. Comput. Chem. 2007, 28, 555-569. [CrossRef]

30. Grimme, S.; Antony, J.; Ehrlich, S.; Krieg, H. A consistent and accurate ab initio parametrization of density functional dispersion correction (DFT-D) for the 94 elements H-Pu. J. Chem. Phys. 2010, 132, 154104. [CrossRef]

31. Grimme, S.; Ehrlich, S.; Goerigk, L. Effect of the damping function in dispersion corrected density functional theory. J. Comput. Chem. 2011, 32, 1456-1465. [CrossRef]

32. Caldeweyher, E.; Bannwarth, C.; Grimme, S. Extension of the D3 dispersion coefficient model. J. Chem. Phys. 2017, 147, 034112. [CrossRef] 
33. Vydrov, O.A.; Van Voorhis, T. Nonlocal van der Waals density functional: The simpler the better. J. Chem. Phys. 2010, 133, 244103. [CrossRef]

34. Sabatini, R.; Gorni, T.; de Gironcoli, S. Nonlocal van der Waals density functional made simple and efficient. Phys. Rev. B 2013, 87, 041108. [CrossRef]

35. Møller, C.; Plesset, M.S. Note on an Approximation Treatment for Many-Electron Systems. Phys. Rev. 1934, 46, 618-622. [CrossRef]

36. Scuseria, G.E.; Henderson, T.M.; Sorensen, D.C. The ground state correlation energy of the random phase approximation from a ring coupled cluster doubles approach. J. Chem. Phys. 2008, 129, 231101. [CrossRef]

37. Bartlett, R.J.; Musiał, M. Coupled-cluster theory in quantum chemistry. Rev. Mod. Phys. 2007, 79, $291-352$. [CrossRef]

38. Marsman, M.; Grüneis, A.; Paier, J.; Kresse, G. Second-order Møller-Plesset perturbation theory applied to extended systems. I. Within the projector-augmented-wave formalism using a plane wave basis set. J. Chem. Phys. 2009, 130, 184103. [CrossRef] [PubMed]

39. Schwartz, C. Importance Angular Correlations between Atomic Electrons. Phys. Rev. 1962, 126, 1015-1019. [CrossRef]

40. Franck, O.; Mussard, B.; Luppi, E.; Toulouse, J. Basis convergence of range-separated density-functional theory. J. Chem. Phys. 2015, 142, 074107. [CrossRef]

41. Grüneis, A.; Marsman, M.; Kresse, G. Second-order Møller-Plesset perturbation theory applied to extended systems. II. Structural and energetic properties. J. Chem. Phys. 2010, 133, 074107. [CrossRef]

42. Kühne, T.D.; Iannuzzi, M.; Del Ben, M.; Rybkin, V.V.; Seewald, P.; Stein, F.; Laino, T.; Khaliullin, R.Z.; Schütt, O.; Schiffmann, F.; et al. CP2K: An electronic structure and molecular dynamics software package-Quickstep: Efficient and accurate electronic structure calculations. J. Chem. Phys. 2020, 152, 194103. [CrossRef]

43. Dovesi, R.; Erba, A.; Orlando, R.; Zicovich-Wilson, C.M.; Civalleri, B.; Maschio, L.; Rérat, M.; Casassa, S.; Baima, J.; Salustro, S.; et al. Quantum-mechanical condensed matter simulations with CRYSTAL. WIREs Comput. Mol. Sci. 2018, 8, e1360. [CrossRef]

44. Barca, G.M.J.; Bertoni, C.; Carrington, L.; Datta, D.; De Silva, N.; Deustua, J.E.; Fedorov, D.G.; Gour, J.R.; Gunina, A.O.; Guidez, E.; et al. Recent developments in the general atomic and molecular electronic structure system. J. Chem. Phys. 2020, 152, 154102. [CrossRef]

45. Förster, A.; Franchini, M.; van Lenthe, E.; Visscher, L. A Quadratic Pair Atomic Resolution of the Identity Based SOS-AO-MP2 Algorithm Using Slater Type Orbitals. J. Chem. Theory Comput. 2020, 16, 875-891. [CrossRef]

46. Perdew, J.P.; Zunger, A. Self-interaction correction to density-functional approximations for many-electron systems. Phys. Rev. B 1981, 23, 5048-5079. [CrossRef]

47. Feyereisen, M.; Fitzgerald, G.; Komornicki, A. Use of approximate integrals in ab initio theory. An application in MP2 energy calculations. Chem. Phys. Lett. 1993, 208, 359-363. [CrossRef]

48. Weigend, F.; Häser, M.; Patzelt, H.; Ahlrichs, R. RI-MP2: Optimized auxiliary basis sets and demonstration of efficiency. Chem. Phys. Lett. 1998, 294, 143-152. [CrossRef]

49. Jung, Y.; Lochan, R.C.; Dutoi, A.D.; Head-Gordon, M. Scaled opposite-spin second order Møller-Plesset correlation energy: An economical electronic structure method. J. Chem. Phys. 2004, 121, 9793-9802. [CrossRef]

50. Furche, F. Developing the random phase approximation into a practical post-Kohn-Sham correlation model. J. Chem. Phys. 2008, 129, 114105. [CrossRef]

51. Eshuis, H.; Yarkony, J.; Furche, F. Fast computation of molecular random phase approximation correlation energies using resolution of the identity and imaginary frequency integration. J. Chem. Phys. 2010, 132, 234114. [CrossRef]

52. Boyd, J.P. Exponentially convergent Fourier-Chebshev quadrature schemes on bounded and infinite intervals. J. Sci. Comput. 1987, 2, 99-109. [CrossRef]

53. Braess, D.; Hackbusch, W. Approximation of $1 / x$ by exponential sums in $[1, \infty]$. IMA J. Numer. Anal. 2005, 25, 685-697. [CrossRef]

54. Braess, D. Nonlinear Approximation Theory; Springer Science \& Business Media: Berlin/Heidelberg, Germany, 2012. 
55. Chai, J.D.; Head-Gordon, M. Long-range corrected double-hybrid density functionals. J. Chem. Phys. 2009, 131, 174105. [CrossRef]

56. Ángyán, J.G.; Gerber, I.C.; Savin, A.; Toulouse, J. van der Waals forces in density functional theory: Perturbational long-range electron-interaction corrections. Phys. Rev. A 2005, 72, 012510. [CrossRef]

57. Toulouse, J.; Zhu, W.; Ángyán, J.G.; Savin, A. Range-separated density-functional theory with the random-phase approximation: Detailed formalism and illustrative applications. Phys. Rev. A 2010, 82, 032502. [CrossRef]

58. Bruneval, F. Range-Separated Approach to the RPA Correlation Applied to the van der Waals Bond and to Diffusion of Defects. Phys. Rev. Lett. 2012, 108. [CrossRef]

59. Brémond, É.; Savarese, M.; Pérez-Jiménez, Á.J.; Sancho-García, J.C.; Adamo, C. Range-Separated Double-Hybrid Functional from Nonempirical Constraints. J. Chem. Theory Comput. 2018, 14, 4052-4062. [CrossRef]

60. Mezei, P.D.; Kállay, M. Construction of a Range-Separated Dual-Hybrid Direct Random Phase Approximation. J. Chem. Theory Comput. 2019, 15, 6678-6687. [CrossRef]

61. Lippert, G.; Hutter, J.; Parrinello, M. A hybrid Gaussian and plane wave density functional scheme. Mol. Phys. 1997, 92, 477-488. [CrossRef]

62. Guidon, M.; Schiffmann, F.; Hutter, J.; VandeVondele, J. Ab initio molecular dynamics using hybrid density functionals. J. Chem. Phys. 2008, 128, 214104. [CrossRef] [PubMed]

63. Sansone, G.; Civalleri, B.; Usvyat, D.; Toulouse, J.; Sharkas, K.; Maschio, L. Range-separated double-hybrid density-functional theory applied to periodic systems. J. Chem. Phys. 2015, 143, 102811. [CrossRef] [PubMed]

64. Civalleri, B.; Zicovich-Wilson, C.; Valenzano, L.; Ugliengo, P. B3LYP Augmented with an Empirical Dispersion Term (B3LYP-D*) as Applied to Molecular Crystals. Crystengcomm 2008, 10. [CrossRef]

65. Kiefte, H.; Penney, R.; Breckon, S.W.; Clouter, M.J. Brillouin scattering studies of isotopic effects in solid ammonia. J. Chem. Phys. 1987, 86, 662-665. [CrossRef]

66. Dulmage, W.J.; Lipscomb, W.N. The crystal structures of hydrogen cyanide, HCN. Acta Crystallogr. 1951, 4, 330-334. [CrossRef]

67. Batchelder, D.N.; Losee, D.L.; Simmons, R.O. Measurements of Lattice Constant, Thermal Expansion, and Isothermal Compressibility of Neon Single Crystals. Phys. Rev. 1967, 162, 767-775. [CrossRef]

68. McConville, G.T. New values of sublimation energy L0 for natural neon and its isotopes. J. Chem. Phys. 1974, 60, 4093-4093. [CrossRef]

69. Endoh, Y.; Shirane, G.; Skalyo, J. Lattice dynamics of solid neon at 6.5 and 23.7 K. Phys. Rev. B 1975, 11, 1681-1688. [CrossRef]

70. Peterson, O.G.; Batchelder, D.N.; Simmons, R.O. Measurements of X-Ray Lattice Constant, Thermal Expansivity, and Isothermal Compressibility of Argon Crystals. Phys. Rev. 1966, 150, 703-711. [CrossRef]

71. Schwalbe, L.A.; Crawford, R.K.; Chen, H.H.; Aziz, R.A. Thermodynamic consistency of vapor pressure and calorimetric data for argon, krypton, and xenon. J. Chem. Phys. 1977, 66, 4493-4502. [CrossRef]

72. Mardirossian, N.; Head-Gordon, M. wB97M-V: A combinatorially optimized, range-separated hybrid, meta-GGA density functional with VV10 nonlocal correlation. J. Chem. Phys. 2016, 144, 214110. [CrossRef]

73. Zhao, Y.; Truhlar, D.G. Design of Density Functionals That Are Broadly Accurate for Thermochemistry, Thermochemical Kinetics, and Nonbonded Interactions. J. Phys. Chem. 2005, 109, 5656-5667. [CrossRef] [PubMed]

74. Grimme, S.; Steinmetz, M. A computationally efficient double hybrid density functional based on the random phase approximation. Phys. Chem. Chem. Phys. 2016, 18, 20926-20937. [CrossRef]

75. Alipour, M. On the opposite-spin to same-spin ratio of absolute and interaction MP2 correlation energy in parameter-free spin-opposite-scaled double hybrids. Chem. Phys. Lett. 2017, 684, 423-426. [CrossRef]

76. Lehtola, S.; Steigemann, C.; Oliveira, M.J.; Marques, M.A. Recent developments in libxc-A comprehensive library of functionals for density functional theory. SoftwareX 2018, 7, 1-5. [CrossRef]

77. Mardirossian, N.; Ruiz Pestana, L.; Womack, J.C.; Skylaris, C.K.; Head-Gordon, T.; Head-Gordon, M. Use of the rVV10 Nonlocal Correlation Functional in the B97M-V Density Functional: Defining B97M-rV and Related Functionals. J. Phys. Chem. Lett. 2017, 8, 35-40. [CrossRef] [PubMed]

78. Goedecker, S.; Teter, M.; Hutter, J. Separable dual-space Gaussian pseudopotentials. Phys. Rev. B 1996, 54, 1703-1710. [CrossRef] 
79. Hutter, J. New Optimization of GTH Pseudopotentials for PBE, SCAN, PBE0 Functionals. GTH Pseudopotentials for Hartree-Fock. NLCC Pseudopotentials for PBE. Latest Commit from 8 August 2019. Available online: https:/ / github.com/juerghutter/GTH (accessed on 30 September 2020)

80. Del Ben, M.; Hutter, J.; VandeVondele, J. Second-Order Møller-Plesset Perturbation Theory in the Condensed Phase: An Efficient and Massively Parallel Gaussian and Plane Waves Approach. J. Chem. Theory Comput. 2012, 8, 4177-4188. [CrossRef]

81. Dunning, T.H. Gaussian basis sets for use in correlated molecular calculations. I. The atoms boron through neon and hydrogen. J. Chem. Phys. 1989, 90, 1007-1023. [CrossRef]

82. Woon, D.E.; Dunning, T.H. Gaussian basis sets for use in correlated molecular calculations. III. The atoms aluminum through argon. J. Chem. Phys. 1993, 98, 1358-1371. [CrossRef]

83. Bernardi, F.. Bivariational procedures for atomic and molecular computations: The transcorrelated method and two new related simplified procedures. J. Phys. France 1973, 34, 373-379. [CrossRef]

84. Chickos, J.S.; Acree, W.E. Enthalpies of Sublimation of Organic and Organometallic Compounds. 1910-2001. J. Phys. Chem. Ref. Data 2002, 31, 537-698. [CrossRef]

85. Gavezzotti, A. Structure and intermolecular potentials in molecular crystals. Model. Simul. Mater. Sci. Eng. 2002, 10, R1-R29. [CrossRef]

86. Chai, J.D.; Head-Gordon, M. Systematic optimization of long-range corrected hybrid density functionals. J. Chem. Phys. 2008, 128, 084106. [CrossRef]

87. Goerigk, L.; Hansen, A.; Bauer, C.; Ehrlich, S.; Najibi, A.; Grimme, S. A look at the density functional theory zoo with the advanced GMTKN55 database for general main group thermochemistry, kinetics and noncovalent interactions. Phys. Chem. Chem. Phys. 2017, 19, 32184-32215. [CrossRef] [PubMed]

88. Santra, G.; Sylvetsky, N.; Martin, J.M.L. Minimally Empirical Double-Hybrid Functionals Trained against the GMTKN55 Database: revDSD-PBEP86-D4, revDOD-PBE-D4, and DOD-SCAN-D4. J. Phys. Chem. 2019, 123, 5129-5143. [CrossRef] [PubMed]

89. Del Ben, M.; Hutter, J.; VandeVondele, J. Electron Correlation in the Condensed Phase from a Resolution of Identity Approach Based on the Gaussian and Plane Waves Scheme. J. Chem. Theory Comput. 2013, 9, 2654-2671. [CrossRef]

Publisher's Note: MDPI stays neutral with regard to jurisdictional claims in published maps and institutional affiliations.

(C) 2020 by the authors. Licensee MDPI, Basel, Switzerland. This article is an open access article distributed under the terms and conditions of the Creative Commons Attribution (CC BY) license (http://creativecommons.org/licenses/by/4.0/). 